\title{
Identification of the Repressive Domain of the Negative Circadian Clock Component CHRONO
}

\author{
Yu Yang $®$, Ning Li, Jiameng Qiu, Honghua Ge ${ }^{\circledR}$ and Ximing Qin *๑ \\ Department of Health Sciences, Institutes of Physical Science and Information Technology, Anhui University, \\ Hefei 230601, Anhui, China; yangyu@ahu.edu.cn (Y.Y.); li1296697397@163.com (N.L.); \\ qiujiameng911@163.com (J.Q.); hhge@ahu.edu.cn (H.G.) \\ * Correspondence: qin.ximing@ahu.edu.cn; Tel.: +86-551-6386-1858
}

Received: 9 March 2020; Accepted: 31 March 2020; Published: 2 April 2020

check for updates

\begin{abstract}
Circadian rhythm is an endogenous, self-sustainable oscillation that participates in regulating organisms' physiological activities. Key to this oscillation is a negative feedback by the main clock components Periods and Cryptochromes that repress the transcriptional activity of BMAL1/CLOCK (defined in the Abbreviations) complexes. In addition, a novel repressor, CHRONO, has been identified recently, but details of CHRONO's function during repressing the circadian cycle remain unclear. Here we report that a domain of CHRONO mainly composed of $\alpha$-helixes is critical to repression through the exploitation of protein-protein interactions according to luciferase reporter assays, co-immunoprecipitation, immunofluorescence, genome editing, and structural information analysis via circular dichroism spectroscopy. This repression is fulfilled by interactions between CHRONO and a region on the C-terminus of BMAL1 where Cryptochrome and CBP (defined in the Abbreviations) bind. Our resultsindicate that CHRONO and PER differentially function as BMAL1/CLOCK-dependent repressors. Besides, the N-terminus of CHRONO is important for its nuclear localization. We further develop a repression model of how PER, CRY, and CHRONO proteins associate with BMAL1, respectively.
\end{abstract}

Keywords: circadian rhythm; CHRONO; repression; BMAL1; TTFL; CRISPR/Cas9

\section{Introduction}

Most organisms have evolved an endogenous circadian clock to adjust their metabolic activities to anticipate daily environmental events, such as day/night switches or temperature changes. In mammals, circadian clocks exist in almost every cell and organ. The clock consists of a transcriptional and translational feedback loop (TTFL) composed by transcription factors brain and muscle arnt-like protein-1 (BMAL1) and circadian locomotor output cycles kaput (CLOCK). BMAL1 and CLOCK form hetero-dimer complexes (B/C complexes in the following text) and then activate transcription of clock controlled genes (ccgs), including genes encoding PER (PER1, and PER2, and PER3) and CRY proteins (CRY1, and CRY2) [1-3]. After translation and post-translational modifications, PERs and CRYs translocate into the nucleus to repress BMAL1/CLOCK complex function [4,5]. With such repression, a full clock cycle is completed. A second loop composed of circadian components, such as rev-erb (I confirm) [6] and ror [7] and their coding products is involved in regulating the transcription of arntl1 gene, which encodes the BMAL1 protein. Other clock genes, such as csnk1d, csnk1e, fblx 21, and $f b l x 3$, contribute to setting the clock speed [8-11] or in entraining the clockwork [12].

Recently, a novel clock gene was discovered using ChIP-seq (and computational analysis) and subsequently analyzed in mammals. It was named ChIP-derived repressor of network oscillator (chrono) [13], or computationally highlighted repressor of the network oscillator [14], or circadian associated repressor of transcription (ciart) [15]. chrono is under control of the clock and its translational 
product, $\mathrm{CHRONO}$ (CHR in the following text), is reported to repress transcriptional activation of $\mathrm{B} / \mathrm{C}$ complexes in a histone deacetylase (HDAC)- dependent manner [13]. chrono deficient mice had longer periods of the locomotor activity, similar to some other negative clock components such as cry, indicating it is a core circadian component [14]. In summary, negative components required to complete a full clock cycle include PERs, CRYs, and CHR as well.

Negative repression is critical to the circadian clock and some means of repression have been identified. First, repressors PERs (PER1 and PER2) were found to remove B/C complexes from chromatin to inactivate transcription in a CRY dependent way [16,17]. In contrast, ChIP assays to map chromatin association by PERs indicated that PER forms large complexes on chromatin during early repression phase $[18,19]$. Studies revealed that PER2 and CRYs bind to different domains of the BMAL1 protein in order to function as repressors [20]. The photolyase superfamily protein CRYs are more potent than PER proteins for repressing B/C complexes [21-23]. Partch lab demonstrated that besides binding to BMAL1, CRY1 also associates with CLOCK as a repressor [24]. During the repression phase, CRYs bind to $\mathrm{B} / \mathrm{C}$ complexes at the $\mathrm{C}$ terminus of BMAL1 on chromatin, sequestering the binding site from transcriptional co-activators, CBP(defined in the Abbreviations)/p300 [23]. Biochemical and cell-based complementation assays suggest that an $\alpha$-helical domain of CRY1 is required for feedback repression and that the $\mathrm{C}$-terminal domain of CRY1 is dispensable for complementation of the circadian clock in cry1/cry2 double knockout cells [22]. Repression of B/C complexes by CHR has been studied and CHR is reported to be similar to CRY1 with regard to circadian clock regulation, via interacting with the BMAL1 C terminus [25]. Anafi et al. reported that CHR functioned at an independent region from (but adjacent to) CRY proteins on the BMAL1 C terminus [14]. However, how repression during the cycle of the circadian clock happens remains unclear, for example, how CHR fulfills its repressor role is elusive. We do not know which region of CHR associates with BMAL1 or the structure of CHR. Thus, to understand how $\mathrm{CHR}$ represses $\mathrm{B} / \mathrm{C}$ complex transcriptional activity, we purified a predicted helical domain of the CHR protein and extended helical domains. Then, we used circular dichroism spectroscopy to confirm secondary structures. We found that the helical domain of CHR is necessary to repress $\mathrm{B} / \mathrm{C}$ complexes in vitro. Immunoprecipitation and Immunofluorescence revealed interactions between the middle area of CHR containing rich $\alpha$-helicesandthe $\mathrm{C}$ terminus of BMAL1, indicating that $\mathrm{CHR}$ repressed $\mathrm{B} / \mathrm{C}$ complexes via its middle area containing an $\alpha$-helix rich region. Interestingly, the N-terminus of CHR contains an unidentified motif (or multiple motifs) that localize CHR into the nucleus. Collectively, characterizations of the helical domain of CHR tell us more about how CHR represses the transcriptional activity of $\mathrm{B} / \mathrm{C}$ complex, compared to the other two negative components (PERs, CRYs).

\section{Materials and Methods}

\subsection{Strains and Constructs}

Polymerase chain reaction (PCR) was used to make constructs described in this study. Optimized sequence coding for CHR-H and CHR-H3were amplified and inserted into the V28-E4 plasmid (from Dr. Ge lab, Anhui University) between NotI and XhoI restriction sites, resulting in a maltose binding protein (MBP) tag and 6XHis tag fused to the $5^{\prime}$ and $3^{\prime}$ ends respectively. MBP-CHRH (or H3) was expressed in the E. coil BL21 (DE3) strain. For the luciferase reporter assay, various versions of chrono as indicated in the text were cloned into pcDNA3.1(+)/Hygro (Invitrogen, Untied States). For immunoprecipitation, sequence of 5Myc-6His-hBMAL1 (5M6HA1) was cloned and inserted into pcDNA3.1 via BmaHI and EcoRI sites (named pcDNA3.1-5M6HA1 afterwards). On the one hand, a series of chrono with cpVenus tag followed sequences were cloned and inserted into pcDNA3.1(+)/Hygro plasmid between HindIII and XhoI sites, on the other hand, we built series of bmal1 with 5XMyc-6XHis-tag, using two EcoRI sites on the mutated pcDNA3.1. 


\subsection{Site-Directed Mutagenesis}

To make L606A/L607A double mutations in the bmall coding region, the whole plasmid pcDNA3.1-5M6HA1 was amplified using a high fidelity Taq polymerase. A pair of complementary primers 5'GGCAGCAATGGCTGTCATCATGAGCGCAGCAGAAGCAGATGCTGGACTGGGTGGC3' and 5'GCCACCCAGTCCAGCATCTGCTTCTGCTGCGCTCATGATGACAGCCATTGCTGCCT3' were used for the mutagenesis. After the amplification, DpnIwas used to digest the template plasmids. Then the product was transformed into the DH5 $\alpha$ competent cells. To obtain the site-directed mutagenesis plasmids, the plasmids from the transformed E.coli colonies were sent to sequencing for identification.

\subsection{Secondary Structure Prediction}

PredictProtein (www.predictprotein.org/), PSIPRED (http://bioinf.cs.ucl.ac.uk/psipred/), and JPred (http://www.compbio.dundee.ac.uk/jpred/) were used for CHR secondary structure prediction and both offered similar results.

\subsection{Nuclear Location Signal Prediction}

NucPred (https://nucpred.bioinfo.se/nucpred/), PredictNLS (https://rostlab.org/owiki/index.php/ PredictNLS), and PSORTII (https://psort.hgc.jp/form2.html), were used for CHR nuclear location signal prediction.

\subsection{Codon Optimization}

Codon usage bias is a general characteristic for all species. The human chronocDNA had a low yield using prokaryotic expression system. We used OPTIMIZER (http://genomes.urv.cat/OPTIMIZER/) and JCat (http://www.jcat.de) to obtain optimized sequences for chrono to express in E. coli, and the full optimized sequence was ordered from General Biosystems, Inc. Anhui Province, China.

\subsection{Protein Expression and Purification}

BL21(DE3) E. coli cells that carry constructs for expression of MBP-CHRH/CHRH3 fusion protein were grown at $37^{\circ} \mathrm{C}, 220 \mathrm{rpm}$ to reach OD values up to 0.6-0.8. Then $0.2 \mathrm{mM}$ IPTG was added to induce expression at $16^{\circ} \mathrm{C}, 150 \mathrm{rpm}$, for 20-24 h. Cell pellets were lysed in $50 \mathrm{mM}$ Tris- $\mathrm{HCl}(\mathrm{pH} 8.0)$, and $200 \mathrm{mM} \mathrm{NaCl}$. Ni-NTA Sefinose ${ }^{\mathrm{TM}}$ Resin (\#C600033, BBI, Shanghai, China) was used for first His tag affinity purification. Then, Hiload 16/600 superdex (200pg) was used as gel filtration purification.

\subsection{Circular Dichroism Spectroscopy}

To verify rich $\alpha$-helix occupation in the secondary structure of $\mathrm{CHR}-\mathrm{H}$, we compared secondary

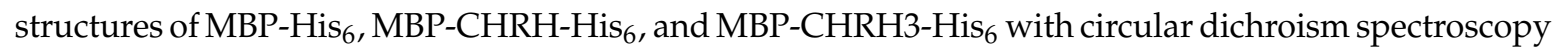
(Biologic/MOS-500). Protein buffer was changed to $50 \mathrm{mM}$ sodium phosphate ( $\mathrm{pH} 8.0$ ), and then the protein sample was diluted to $0.1 \mathrm{mg} / \mathrm{mL}$ and clarified using by centrifugation $\left(12,000 \mathrm{rpm}, 4^{\circ} \mathrm{C}, 10 \mathrm{~min}\right)$ before measurement. Each CD spectrum was collected at room temperature, with a scan rate of $1 \mathrm{~nm} / \mathrm{s}$; scanning increment, $0.5 \mathrm{~nm}$; spectral bandwidth, $1.0 \mathrm{~nm}$; from 190-260 nm for each measurement. The spectra represent the average of 3 scans with the bassline subtracted from analogous conditions.

\subsection{Luciferase Reporter Assay}

HEK-293T cells were grown in DMEM (SH30022, Hyclone/GE, United States) supplemented with $10 \%$ fetal bovine serum (P30-3302, PAN-Biotech, Germany) plus $100 \mathrm{IU} / \mathrm{mL}$ penicillin and $0.1 \mathrm{mg} / \mathrm{mL}$ streptomycin (SV30010, Hyclone/GE, United States) at $37^{\circ} \mathrm{C}, 5 \% \mathrm{CO}_{2}$. Approximately $3 \times 10^{5}$ cells were seeded in each well of a 6-well plate 1 day before transfection. When cells reached $70 \% \sim 90 \%$ confluency, $100 \mathrm{ng}$ of the firefly luciferase reporter $\mathrm{pP}_{\mathrm{PK} 2}:: F l u c, 40 \mathrm{ng}$ of the renilla luciferase reporterp $\mathrm{P}_{\mathrm{CMV}}:: \mathrm{Rluc}$, $250 \mathrm{ng}$ of a hamster Bmal1 construct, and $250 \mathrm{ng}$ of a mouse Clock construct and $500 \mathrm{ng}$ of mouse Per2 (or human CHR, CHR-N, CHRNH, CHR-H, CHRH2, CHRH3, CHRH4, CHRHC, and CHR-C) 
were co-transfected into HEK 293T by using Lipo6000" ${ }^{\mathrm{TM}}$ Transfection Reagent (C0526, Beyotime, Beijing, China). The constant amount of DNA (1100 ng/well) was adjusted with pcDNA3.1(+)/Hygro vector as a carrier, and we measured single luminescence. Twenty-four hours after transfection, cells were washed three times with ice-cold PBS (SH30256.01, Hyclone/GE, United States), then processed with a TransDetect Double-Luciferase Reporter Assay Kit (FR201, Transgen Biotech, Beijing, China), and measured by SpectraMax Paradigm Multi-Mode Microplate Reader (Molecular Devices, Silicon Valley, United States). Each construct was examined at least for three independent times.

\subsection{Western Blotting and Immunoprecipitation}

Mouse antibodies against Myc-tag (M185-3L/M047-3, MBL, Nagoya, Japan), rabbit antibody against GFP-tag (D110008, BBI, Shanghai, China) and sheep antibody against Tubulin (ATN02, Cytoskeleton, Denver, United States) were subjected to Western blot according to the manufacturer's protocol. For IP, HEK-293T cells transfected with the desired plasmids by using Attractene Transfection Reagent (301005, QIAGEN, Hilden, Germany), according to the fast-forward protocol, were lysed in IP buffer (10 mM Tris pH 7.5, $150 \mathrm{mM} \mathrm{NaCl}, 1 \mathrm{mM}$ EDTA, 1\% Triton X-100, 0.1\% sodium deoxycholate, $1 \mathrm{mM}$ PMSF and Roche complete EDTA free protease inhibitor cocktail). After quantification by using BCA Protein Assay Kit (P0012, Beyotime, Beijing, China), lysates were precleared with Protein G Agarose (P2009, Beyotime, Beijing, China) and then immunoprecipitated with mouse anti-Myc antibodies. After washing five times, the precipitates were resuspended in the 5XSDS loading dye, boiled for $5 \mathrm{~min}$, and run on a 12.5\% SDS-PAGE gel followed by Western blot analysis. Immunoreactive bands were detected by Typhoon laser scanners (FLA-9500, GE, United States).

\subsection{Immunofluorescence and Localization}

For IF (immunofluorescence), Hela cells were grown in DMEM supplemented with $10 \%$ FBS plus $100 \mathrm{IU} / \mathrm{mL}$ penicillin and $0.1 \mathrm{mg} / \mathrm{mL}$ streptomycin at $37{ }^{\circ} \mathrm{C}, 5 \% \mathrm{CO}_{2}$. A sterile cover glass was placed into each well of a 6-well plate, and then $2 \times 10^{5}$ cells per well were seeded 1 day before transfection. When cells reached 70\% 90\% confluency, they were transfected with Lipo6000 ${ }^{\mathrm{TM}}$ Transfection Reagent with various combinations of the following constructs (all in pcDNA3.1 (+)/Hygro background): 1,250 ngCHR-cpVenus or CHRH3-cpVenus and 1,250 ng 5XMyc-6XHis-hBMAL1, 5XMyc-6XHis-hBMAL1-C, or 5XMyc-6XHis-hBMAL1-Cs. Twenty-four hours after transfection, cells were washed three times with ice-cold PBS and fixed in $4 \%$ paraformaldehyde for $30 \mathrm{~min}$ at room temperature. Then cells were permeabilized and blocked with $0.3 \%$ TritonX-100 and 5\%FBS in PBS for $1 \mathrm{~h}$ at room temperature follow by sequential washing with PBS, and were subjected for incubation using Myc-tag antibody (ab32, UK, abcam, diluted in PBS containing 3\%BSA) for 1h at room temperature. By following three 5 min PBS washes, cells were immune-stained by Cy3-conjugated antibody (D110172, BBI, Shanghai, China, diluted in PBS containing 1\%BSA/0.3\%TritonX-100) for $30 \mathrm{~min}$ at room temperature. After last three times PBS wash, cells were embedded in Mounting Medium, Antifading (with DAPI, S2110, Solarbio). Analyses and documentation were done with immunofluorescence microscopy (TCS SP5, Leica, Germany). For localization assessment, Hela and NIH 3 T3 cells were cultured in the same media, and a sterile cover glass was placed into each well of a 6-well plate, and then $2 \times 10^{5}$ cells per well were seeded 1 day before transfection. When cells reached $70 \% \sim 90 \%$ confluency, they were transfected with Lipo6000 Transfection Reagent with series of cpVenus fused truncations of CHR (all in pcDNA3.1 (+)/Hygro background): 2500ngCHR-cpVenus/cpVenus-CHR/CHRN-cpVenus/CHRH-cpVenus/CHRC-cpVenus or EGFP. Twenty-four hours after transfection, cells were washed three times with ice-cold PBS and fixed in $4 \%$ paraformaldehyde for $30 \mathrm{~min}$ at room temperature. After three times PBS washes, cells were embedded in Mounting Medium, Antifading and documented by immunofluorescence microscopy. 


\subsection{Chrono Knock Out in U2-OS Cells}

chrono ${ }^{-/}$bmal $^{\text {Luc }}$ cell line was generated using the CRISPR/Cas 9 system. Oligonucleotides specific for the target sites of chrono gene loci were designed using the Optimized CRISPR Design tool (www.genomeengineering.org/). The highest quality score sgRNA (CCGCTGCAGGCATCGATCGA) targeted to the exon 1 of chrono (on-target locus: chr1:+150255862) was chosen to insert into the expression vector pX459 (pSpCas9\{BB\}-2A-Puro was a gift from Feng Zhang, Addgene plasmid \# 48139). $24 \mathrm{~h}$ after transfection, cells were screened with $2 \mu \mathrm{g} / \mathrm{mL}$ puromycin. Cell line clones were screened by the limiting dilution method.

\subsection{Bioluminescence Recoding and Data Analysis}

$2 \times 10^{5} \mathrm{U} 2$-OS reporter cells were seeded in $35 \mathrm{~mm}$ dishes the day before synchronization. On the next day, cells in each dish were synchronized with $2 \mathrm{~h}$ treatment of $200 \mathrm{nM}$ dexamethasone (dissolved in DMSO) and recorded in a LumiCycle as described previously [26]. Bioluminescence data were analyzed with the LumiCycle analysis program (Actimetrics, USA) to obtain circadian parameters such as period and amplitude.

\section{Results}

\subsection{The Helical Domain of CHR Represses the Transcriptional Activity of B/C Complexes}

Since there were neither homologous proteins nor similar protein structures being reported, assessment of CHR amino acid sequences was used to predict its secondary structure. Predict Protein [27] and PSIPRED [28] were used to predict that CHR contains an $\alpha$-helix rich region in the middle (Figure 1A) and both ends of CHR are flexible regions. Thus, CHR can be separated into three domains: a N-terminal domain (CHRN, 1-110), a helical domain (CHRH, 111-196), and a C-terminal domain (CHRC, 197-385).

CHR was identified as a circadian clock component that functions as a transcriptional repressor of $\mathrm{B} / \mathrm{C}$ complexes $[13,14]$. However, details of the binding regions of $\mathrm{CHR}$ to $\mathrm{B} / \mathrm{C}$ complexes, in other words the functional domains of CHR, are unknown. Then, we searched the area of CHR that functions as an inhibitor to the transcription activator $\mathrm{B} / \mathrm{C}$ complexes using the luciferase reporter assay. Based on the prediction, luciferase reporter assays were used to test domain effects including CHRN, $\mathrm{CHRH}$ and CHRC with respect to transcription activation by the B/C complexes in HEK293 cells. In this mammalian reporter system, both mPeriod-2 (mPer2) and full-length CHR as positive controls repressed the $\mathrm{B} / \mathrm{C}$ complexes using a reporter $\mathrm{pP}_{\mathrm{PK} 2}:$ :Fluc (pR2.8) that contains E-box (CACGTG) elements in the promoter region and a reporter $\mathrm{pP}_{\mathrm{CMV}}:$ :Rluc (R-luc) as the internal reference [29]. However, $\mathrm{CHRN}, \mathrm{CHRH}$, or CHRC domain alone was unable to repress transcription activation by the $\mathrm{B} / \mathrm{C}$ complexes (Figure 1B), which indicated the repressive domain was larger than any individual part. Therefore, we performed the same assay by combing individual parts (CHRNH and CHRHC represent the combinations of CHRN, $\mathrm{CHRH}$, and CHRC) and found CHRHC can repress the transcription activity as strongly as the PER2 protein (Figure 1C). Furthermore, a series of the extended constructs, CHRH2 (111 262), CHRH3 (111 264), and CHRH4 (111 298), were made on the basis of the CHRH and CHRHC and tested by the luciferase reporter assay. As the data indicated, CHRH3 and CHRH4 prevented the activation by the $\mathrm{B} / \mathrm{C}$ complexes, while $\mathrm{CHRH} 2$ was not able to repress the activation (Figure 1D). Thus, the functional domain of $\mathrm{CHR}$ protein that represses $\mathrm{B} / \mathrm{C}$ complexes lies in the middle of CHR is predicted to be an $\alpha$-helix rich region. 
A

B

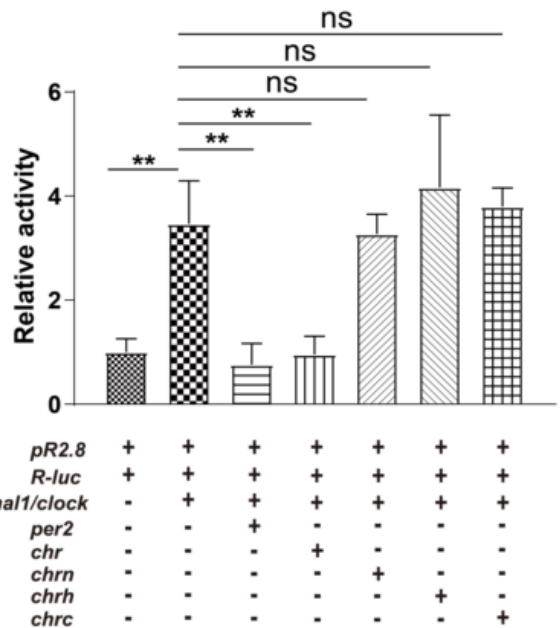

C

D
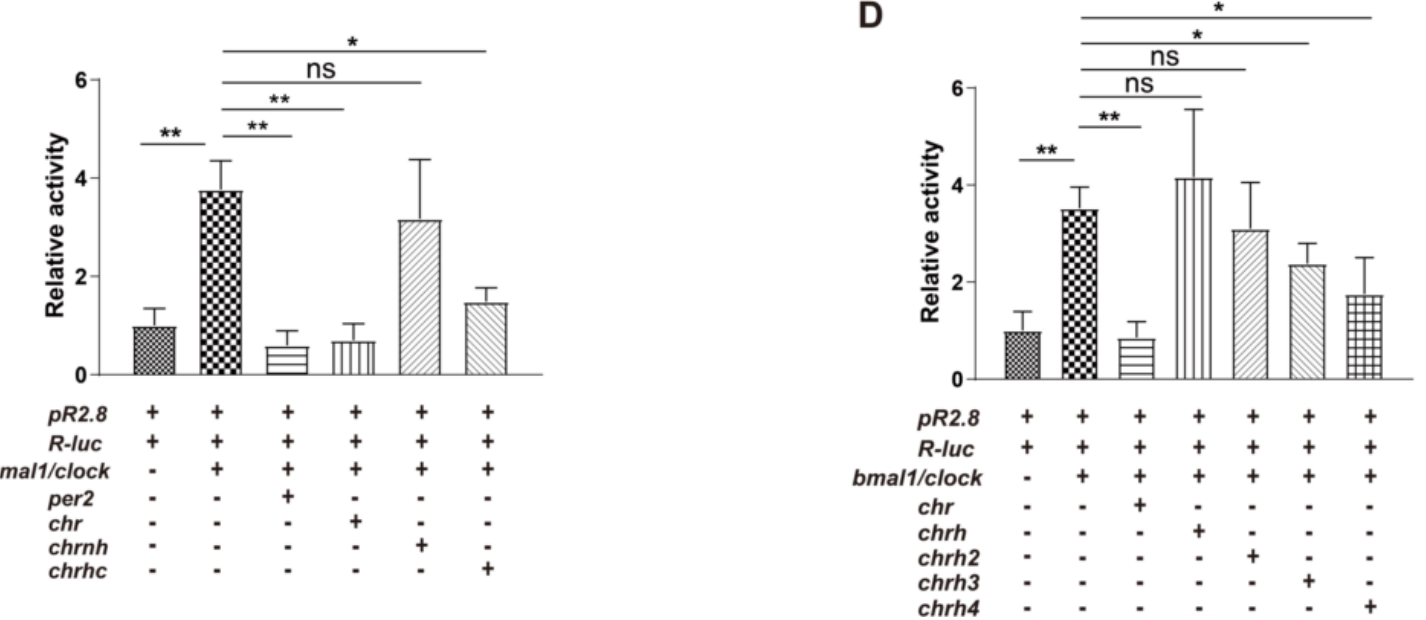

Figure 1. CHR repression domain that contains an $\alpha$-helix rich region. (A) Predicted secondary structures show an $\alpha$-helix rich region in the middle of $\mathrm{CHR}$, with flexible regions in the $\mathrm{N}$ - and $\mathrm{C}$ termini. Pie charts show that CHR containing a large proportion of flexible composition and a lot of regions are exposed. (B) Based on predicted secondary structures, CHR was divided into 3 separate parts: CHRN (1-110), CHRH (111-196), and CHRC (197-385). In the classical luciferase reporter assay which uses $\mathrm{B} / \mathrm{C}$ complexes to activate an E-box containing promoter (pR2.8 in this experiment), each individual part of CHR lost the repressive activity of CHR. (C) However, the combination of the predicted helix region plus the C-terminus (CHRHC, 111-385) is able to function as a repressor, rather than the combination of the helix region plus the $\mathrm{N}$-terminus (CHRNH, 1-196). (D) Luciferase assays indicate that $\mathrm{CHRH}$ plus extended regions at its C-terminus (CHRH3, 111-264 and CHRH4, 111-298) could repress the transcription activation by B/C complexes. Since CHRH3 is 2 amino acids longer than $\mathrm{CHRH} 2$ that did not perform the repressive function, this indicates that the helix containing $\mathrm{H} 3$ is the minimal domain that functions as a repressor to $\mathrm{B} / \mathrm{C}$ complexes. At least three independent experimental repeats were done for each luciferase reporter assay. Graphpad Prism 5 was used to generate graphs/plots and perform statistical analysis (2-tailed unpaired $\mathrm{t}$-test). ns, no significance; ${ }^{*} p<0.05 ;{ }^{* *} p<0.01$.

\subsection{Secondary Structure of MBP-CHRH/CHRH3 (CHRH or CHRH3 fused with MBP) Measured by CD Spectroscopy}

To better understand the repression domain, we went on to explore the structure of CHR protein. We first attempted to acquire its tertiary structures through protein crystallization. We tried different tags (GST, 6XHis, or MBP) and series of truncations to purify CHR proteins. Eventually, we chose $\mathrm{MBP}$ as a tag to purify truncated CHR proteins. However, we were not successful to get information of CHR's tertiary structure through crystallization by now. Based on our luciferase reporter assays, 
we decided to validate the predicted secondary structure by CD spectroscopy. MBP tagged CHRH and CHRH3 were chosen to carry out the CD experiments. The yield of MBP-CHRH was $\sim 40 \mathrm{mg}$ per liter culture, while that of MBP-CHRH3 was merely $2 \mathrm{mg}$ per liter which may be due to the increase of flexible region (Figure 2A,B). According to the in silico prediction, $\mathrm{CHRH}$ is $\alpha$-helix rich, and the component of CHRH3's elongated part is mainly flexible (Figure 2C). Based on the data from circular dichroism spectra (Figure 2D), we analyzed the secondary structure content via K2D, a neural network method [30]. According to the analysis (Figure 2E), proportion of alpha helices increased significantly when MBP fused with $\mathrm{CHRH}$ and CHRH3. These analyses are consistent with our secondary structure prediction that $\mathrm{CHRH}$ is an $\alpha$-helix rich region.

A

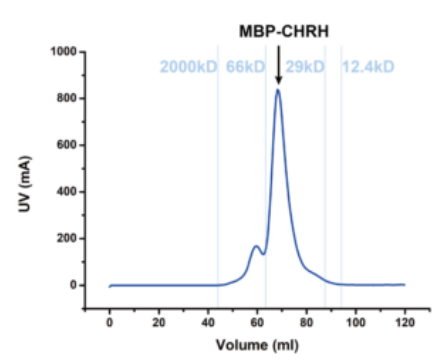

B

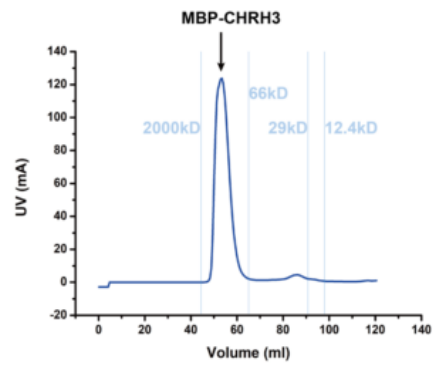

C
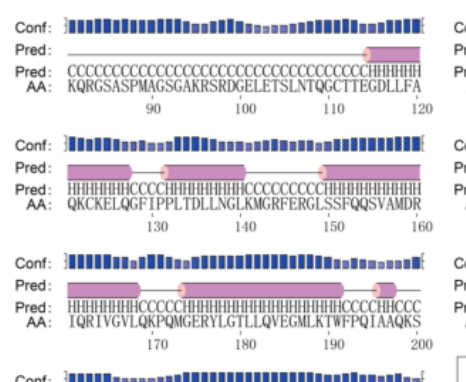

Conf:
Pred:
Pred:

Pred: $\overline{c c c c c c c c c c c c c c c c c c c c c c c c c c c c c c c c c c c c c c c c c c c c c}$
M: SLGGGKHQLTKHFPSHISDSASSPASPIEKMDQTQLCGH.
210

$210 \quad 220 \quad 230 \quad 20$

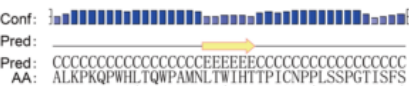

$250 \quad 260$ CHRH(111 196)

CHRH3(111 264)
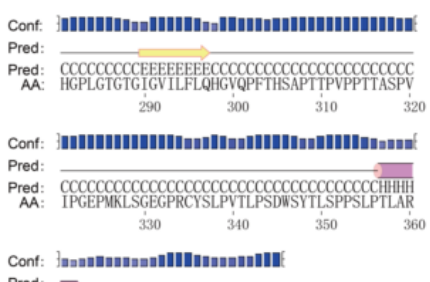

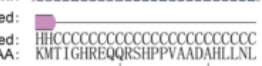

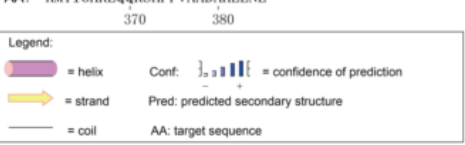

$=$ coll AA: target sequence

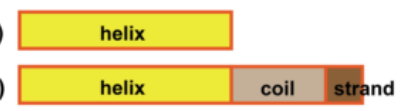

E

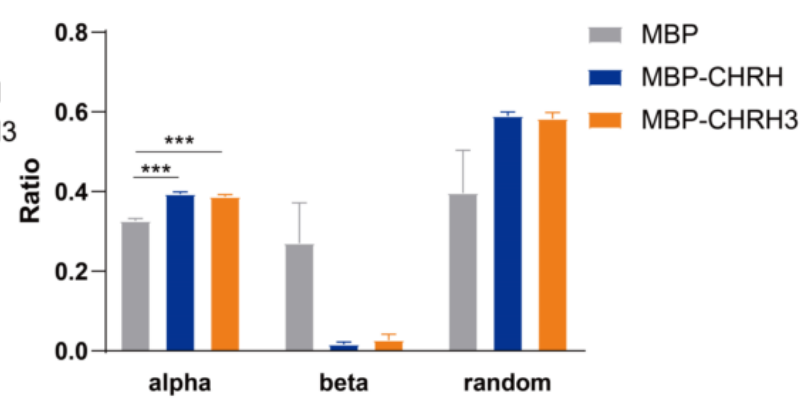

Figure 2. CD spectra ofmaltose binding protein (MBP), MBP-CHRH, and MBP-CHRH3.MBP-CHRH (A) and MBP-CHRH3 (B) purified by gel filtration chromatography. According to the calibration (molecular weight standards were labeled with light blue lines), MBP-CHRH3 has a larger molecular weight than MBP-CHRH. We collected proteins at the peak area for subsequent experiments. (C) According to the result of secondary structure prediction from PSIPRED, CHRH, from 111 196, is mainly composed of $\alpha$-helix motifs; CHRH3, from 111 264, has extra coils and $\beta$-strand motifs in comparison to CHRH. (D) Curves of the CD spectra and (E) comparisons of the secondary structure contents between MBP, MBP-CHRH, and MBP-CHRH3 proteins. MBP-CHRH and MBP-CHRH3 have similar contents of helixes, while MBP has less helical contents. At least three independent experimental repeats were done for each variant. Graphpad Prism 5 was used to generate graphs/plots and perform statistical analysis (2-tailed unpaired t-test). ${ }^{* * *} p<0.001$. 


\subsection{The Helix-Rich Domain is Necessary for CHR/BMAL1 Interaction}

In light of luciferase reporter assays, we sought to confirm the interaction between CHR and BMAL1 using co-immunoprecipitation (co-IP). HEK-293T cells were co-transfected with BMAL1 fused to a 5Myc-6His tag (5M6HA1, short for 5Myc-6His-BMAL1) on the N-terminus and CHR fused to $\mathrm{cpVenus}$ on the C-terminus (CHRV, short for CHR-cpVenus). The C-terminus fusion with a cpVenus tag did not interfere with its cellular localization. In consistent with previous reports that CHR interacts with BMAL1 [13-15], CHRV could be co-immunoprecipitated from HEK-293T cells lysates together with BMAL1 proteins using an anti-Myc antibody (Figure 3A). Subsequently, we tested the possible direct interactions between BMAL1 and a series of CHR constructs as shown in Figure 1. In line with previous luciferase reporter assays, we found that $\mathrm{CHRN}, \mathrm{CHRH}$, or $\mathrm{CHRC}$ alone fused to cpVenus (CHRNV, CHRHV, or CHRCV) was not able to interact with 5M6HA1 (Figure 3B). Again, in agreement with the reporter assays, CHRHC-cpVenus (CHRHCV) was co-immunoprecipitated with BMAL1using anti-Myc antibodies, indicating that the CHRHC region is necessary for BMAL1/CHR interaction (Figure 3C). Moreover, in the same system, shorter CHRHC truncated variants (CHRH3 and CHRH4) could also be immunoprecipitated by anti-Myc antibodies from extracts of HEK-293T cells co-transfected with 5M6HA1 (Figure 3D,E), while CHRH2, merely two amino acids shorter than $\mathrm{CHRH} 3$, could not (Figure 3F). These results signify that the middle region of CHR (111 264) is the minimum area for BMAL1/CHR interaction. In summary, the middle region of CHR that contains a highly helical domain is vital for CHR'sfunction as a transcriptional repressor.

A

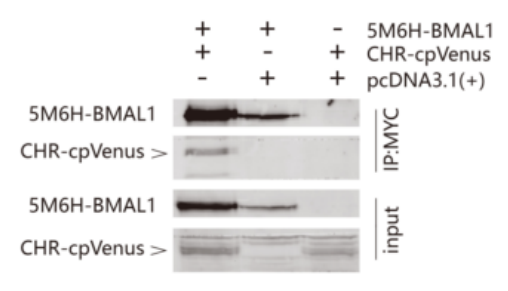

C

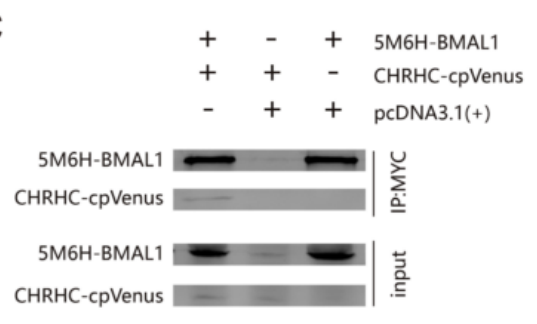

E

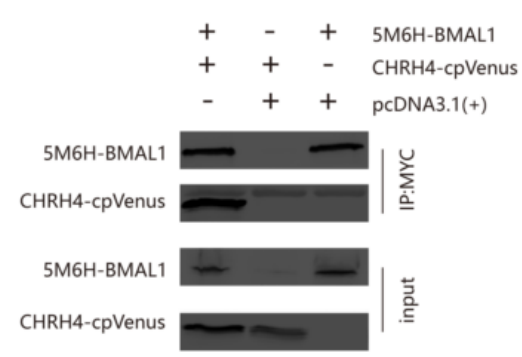

B

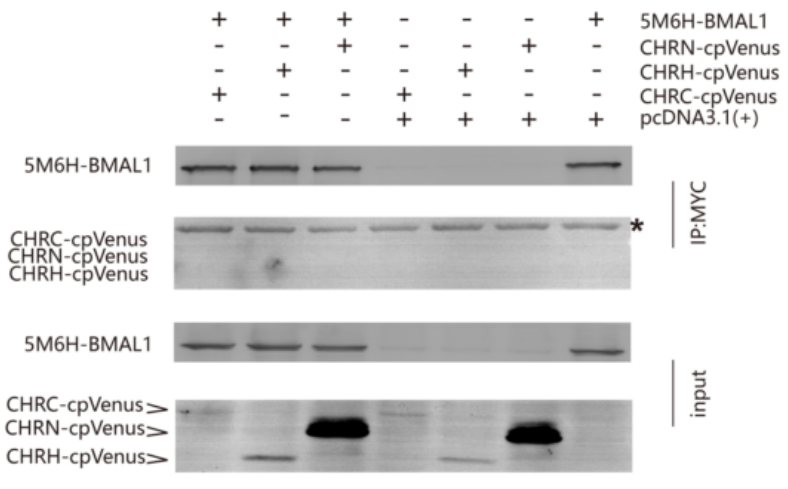

D

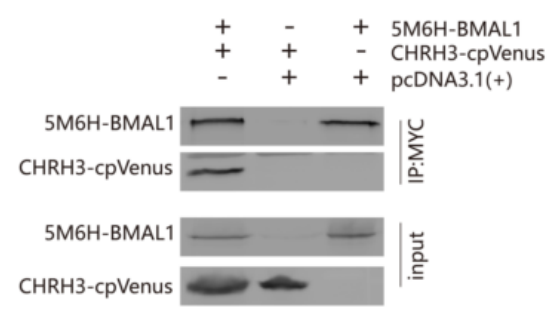

F

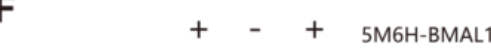

++- CHRH2-cpVenus

$-++\operatorname{pcDNA3.1(+)}$

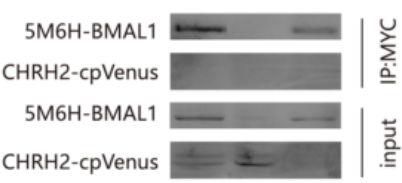

Figure 3. Co-immunoprecipitation (Co-IP) assays indicate that the helix-rich domain is necessary for CHR/BMAL1 interaction. (A) Full-length CHR fused with cpVenus could be immunoprecipitated by 
full-length BMAL1 fused with a 5Myc-6His-tag when both constructs were co-transfected in HEK 293T cells. The cellular extracts were immunoprecipitated with anti-Myc antibodies and detected by using anti-GFP antibodies. (B) CHRN, CHRH, and CHRC were not able to be immunoprecipitated by full-length BMAL1 when they were individually co-transfected with BMAL1 in HEK 293T cells. Consistent with the luciferase reporter assays, CHRHC. (C) and its shortened truncations, CHRH3 (D) and CHRH4 (E), were immunoprecipitated by 5M6H-BMAL1when they were individually co-transfected with BMAL1 in HEK 293T cells. (F) CHRH2, with two amino acids deleted at CHRH3's C-terminus, failed to be immunoprecipitated by 5M6H-BMAL1.At least two independent experimental repeats were done for each assay. Asterisk mark in panel B designates signals of the heavy chainof IgG.

\subsection{The C-Terminus of BMAL1 is Responsible for CHR Binding}

Previously, a region (514 594) of BMAL1 adjacent to the CRY1 interacting terminus was reported to interact with the full-length CHR (14). This interaction disrupts the binding between B/C complexes and CRE binding protein (CBP) or p300, the transcriptional co-activator of circadian clock pathways [14]. However, more convincing evidence from immunoprecipitation or other reliable interaction assays is still lacking. To better understand how CHR interacts with BMAL1 to repress the activity of B/C complexes, we decided to confirm the interaction between CHR and BMAL1C (C-terminus of BMAL1). However, in our data, 5Myc-6His-hBMAL1-C (5M6HA1C, 514 594) failed to co-immunoprecipitate with the full-length CHR protein (Figure 4A). We also carried out immunofluorescence (IF) assays to test their co-localizations (Figure $4 \mathrm{H}$ ). As a transcriptional repressor, exogenously expressed CHRV is located in the nuclei, as detected by the fused fluorescent protein. Our IF assay using anti-Myc antibodies indicated that BMAL1-C is located in the cytoplasm. The merged image implied that CHRV and 5M6HA1-Cwere not co-localized together (Figure 4H), suggesting that they do not interact with each other. This result support our co-IP results (Figure 4A). Under such circumstances, we believed that there is no sufficient evidence that the right domain of BMAL1 C-terminus has been identified for BMAL1/CHR interaction. Since we knew that the last 43 amino acids of BMAL1 are required for transcriptional activation [23] and CBP also binds to it [25], we hypothesized that CHR disturbed CBP's co-activation by binding to the last 43 aa of BMAL1(583 626) as well. To test our hypothesis, firstly, we tested whether CHR interacted with the C-terminus of BMAL1. Indeed, 5Myc-6His-hBMAL1-N (5M6HA1-N, 1 490) was not capable of co-immunoprecipitating with CHRV together from extracts of HEK-293T cells (Figure 4B), while the 5Myc-6His-hBMAL1-C longer version (5M6HA1Cl, 490 626) succeeded (Figure 4C). Then, we used 5Myc-6His-hBMAL1-C shorter version (5M6HA1Cs, 579 626), containing the last 43 aa, to pull CHRV down, and it also succeeded in precipitating the CHRV protein (Figure 4D). CRY1 and CBP has been shown to compete for BMAL1 TAD domain binding [31,32], and the IxxLL motif in the TAD $\alpha$-helix is the major binding site mediating the interaction with CRY1and CBP [25]. Thus, we constructed L606A and L607A double mutant 5M6HA1 (mut5M6HA1), where the IxxLL motif was destroyed [25], to examine whether CHR competes with CBP by binding to TAD domain directly. We found the interaction between BAML1 and CHR was abolished when the IxxLL motif was disrupted (Figure 4E). In other words, CHR may have a competition with CBP for BMAL1 TAD binding. Since CHRH3 retains the repression function of CHR (Figure 1D), we used 5M6HA1Cs to pull CHRH3V down. Although CHRH3V can be precipitated, the CHRH3/BMAL1Cs interaction is weaker compared with the interaction between CHR and BMAL1Cs (Figure 4D,F). It is possible that the functional domain may need extra parts to stabilize the interaction. Therefore, we tested the interaction between 5M6HA1Cs and CHRH4V, which is able to repress the transcriptional activity evoked by B/C complexes and be immunoprecipitated by BMAL1 as well (Figures 1D and 3E). The CHRH4 showed a stronger binding with the C-terminus of BMAL1, compared to CHRH3 (Figure 4F,G). In summary, we suggested that $\mathrm{CHRH} 3$ is the minimal domain to exercise as a repressor via directly binding to BAML1-Cs, and the coils adjacent to its C-terminus might be an additional stabilizing part to maintain the binding. Similarly, the co-localization between CHR and the C-terminus of BMAL1 via IF supports 
the idea that they interact with each (Figure $4 \mathrm{H}$ ). Consistently, the short construct CHRH3 showed less co-localization, compared to the full-length CHR (Figure 4H, bottom VS. middle). Thus, the repressing domain, $\mathrm{CHRH} 3$, is important for interactions between $\mathrm{CHR}$ and $\mathrm{B} / \mathrm{C}$ complexes through the TAD domain at BMAL1's C-terminus.

A

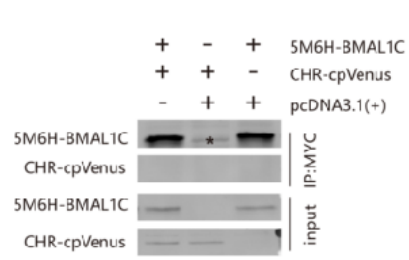

D

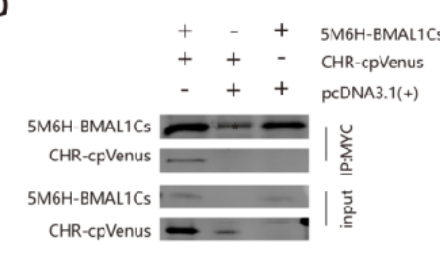

G

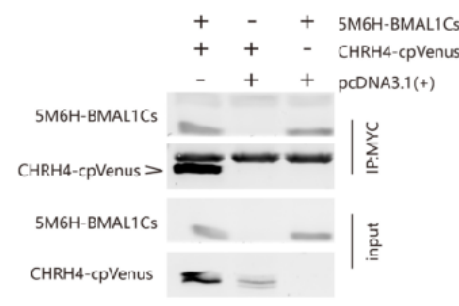

B

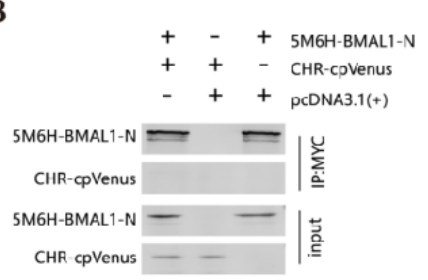

E

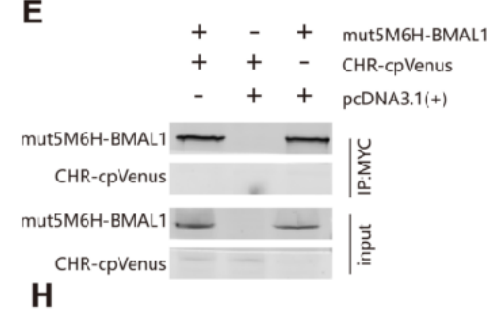

$\mathrm{H}$

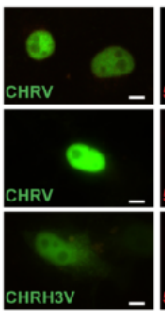

C

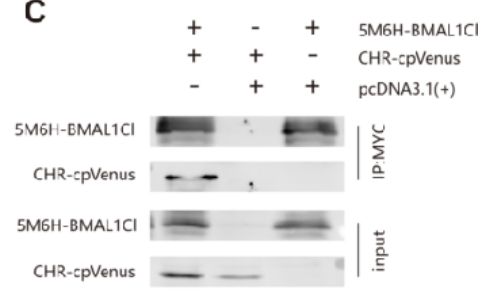

$\mathbf{F}$

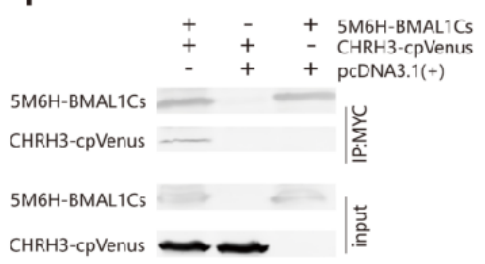

СнRн3-срVелич

Figure 4. Co-IP and IF (immunofluorescence) suggest that the C-terminus rather than other regions of BMAL1 interacts with CHR. (A) The unique region of BMAL1 reported by previous study (14), from 514 to 594, could not pull down CHR in HEK293T cell extracts. (B) The N-terminus of BMAL1 is not able to be co-immunoprecipitated with full-length CHR either when both were co-transfected in HEK293T cells. (C) However, a construct of the intact C-terminus of BMAL (from 490 to 626) named as BMAL1-Clong (BMAL1Cl) is able to precipitate full-length CHR in HEK293T cells. (D) BMAL1-C short (BMAL1Cs, from 579 to 626), the distal C-terminal domain of BMAL1, is sufficient to immunoprecipitateCHR from HEK293T cell extracts when both BMAL1Cs and CHR were co-transfected. (E) Via disruption of the IxxLL motif in the TAD domain at BMAL1's distal C-terminus via Leucine-to-Alanine mutations at 606 and 607 sites, full-length CHR was not able to be precipitated using anti-Myc antibodies that successfully pulled down 5M6H tagged BMAL1 proteins. (F) BMAL1Cs could weakly precipitate with the minimum repressing domain of CHR (CHRH3) using anti-Myc antibodies, which agrees to the luciferase reporter assays. (G) In comparison to $\mathrm{CHRH} 3$, more $\mathrm{CHRH} 4$ was precipitated by BMAL1Cs. (H) In line with these IP results, immunofluorescence (IF) confirmed the interactions between BMAL1-Cs and full-length CHR and the minimum repressor $\mathrm{CHRH} 3$, while CHR failed to co-localize with BMAL1-C (514 594). Bar $=10 \mu \mathrm{m}$. Note that an unspecific band appeared when the short forms of BMAL1 C-terminus was used in the IP assays. Asterisk marks in panels (A) and (D) designate unspecific precipitated products using anti-myc antibodies that ran at the same position with 5M6H-BMAL1C or short version of BMAL1C. A few dots in panel C appeared due to contamination during the immunoblotting process. At least two independent experiments were carried out as repeats.

\subsection{CRISPR Knocking-Out of CHR Disturbs the Circadian Timekeeping}

As one of the core components of mammalian molecular circadian clock, BMAL1's C-terminal TAD domain determines circadian period [25]. Since CHR interacts with BMAL1's TAD domain, we proposed that $\mathrm{CHR}$ also contributes to generating the $24 \mathrm{~h}$ period. Previous studies have 
demonstrated that knocking-out chrono in animals could lengthen the circadian period [13-15], which agrees with our proposal. Nevertheless, it is unclear whether $\mathrm{KO}$ of chrono in cultured cells would interfere with the circadian period. Using the CRISPR/Cas9 system [33], the highest score of a 20-nt guide sequence targeting exon 1 of chrono gene was selected as the sgRNA, which is described in the M\&M. The chrono gene was knocked out in U2-OS cells which harbor a luciferase reporter under the control of bmal1 promoter, generating chrono $^{-/-}$bmal1 ${ }^{\text {Luc }}$ U2-OS cells. We PCR-amplified the target region from chrono ${ }^{-/}$bmal1 $^{\text {Luc }}$ genomic DNA, followed by sequencing. The results showed that both chrono alleles were modified, with one allele losing $4 \mathrm{bp}$ and the other allele having $1 \mathrm{bp}$ insertion at the Cas9 cleavage site (Figure 5A). These frameshift mutations created premature translation stop codons, which eventually knocked out chrono in U2-OS cells (Figure 5B). Compared to the wild-type U2-OS cells (WT, $22.18 \pm 0.068 \mathrm{~h}$ ), chrono ${ }^{-/}$bmal1 $^{\text {Luc }}$ cells $(24.47 \pm 0.453 \mathrm{~h}$ ) exhibited lengthened circadian period by $\sim 2 \mathrm{~h}$ (Figure 5C,D). Our cell-based results were consistent with previous animal studies. Overall, these data suggested that CHR plays a role in maintaining the $24 \mathrm{~h}$ periodicity via interacting with the C-terminal TAD domain of BMAL1.

A

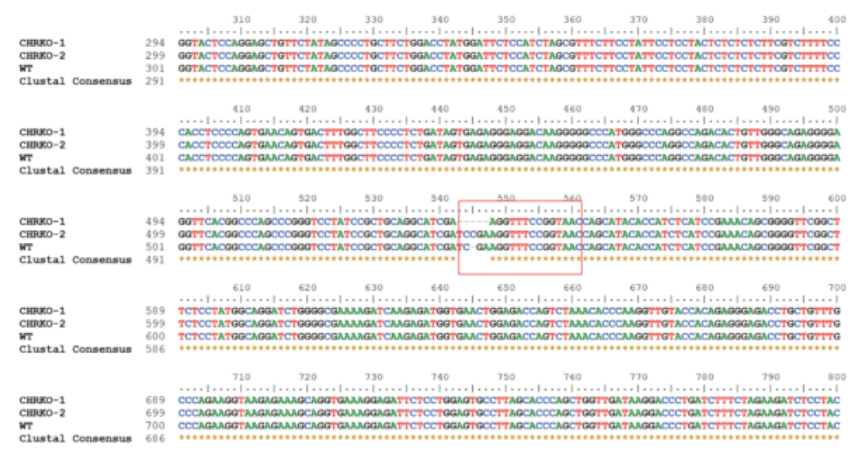

B

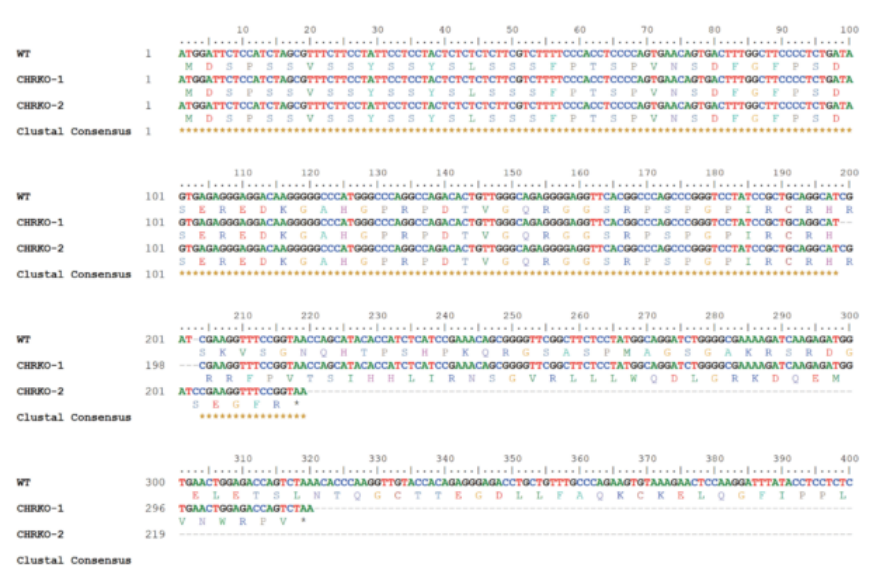

C

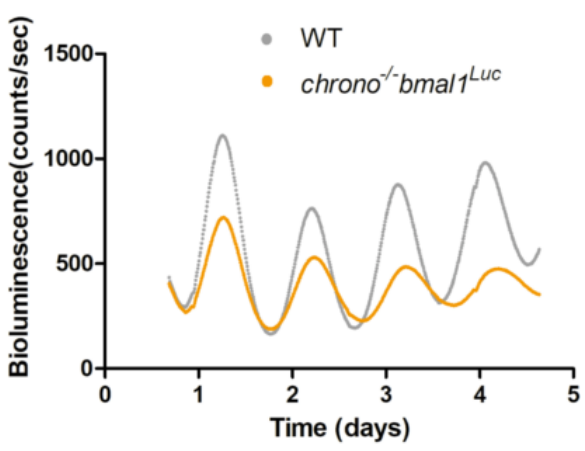

D

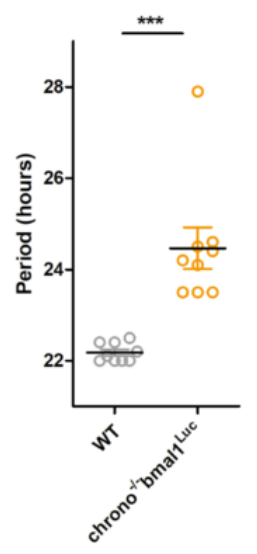

Figure 5. chrono deletion gives rise to longer period in U2-OS cell line. (A) Sequence alignment between wild-type and chrono $^{-/-}$bmal1 $^{\text {Luc }}$ cells showed that the knockout cell line is heterozygous with four bases deleted in one allele and one base insertion in the other. (B) These indels caused early termination of translation through sequence analysis. Corresponding amino acid sequences are presented underneath the nucleotide sequences. Asterisk marksindicate the stop codons caused by the indels. (C) Representatives of raw bioluminescence data from U2-OS cells expressing BMAL1:dLUC reporter are plotted for both $\mathrm{WT}$ and chrono $^{-/}$bmal1 ${ }^{L u c}$ cell lines. (D) Period measured by Lumi Cycleluminometer is plotted using nine repeats from each cell line. Graphpad Prism 5 was used to generate graphs/plots and perform statistical analysis (2-tailed unpaired t-test). ${ }^{* * *} p<0.001$. 


\section{6. cpVenus Fused to the N-Terminus of CHR Interferes with Its Nuclear Localization}

We have identified the domain of CHR that interacts with BMAL1 to repress the transcription activation of $\mathrm{B} / \mathrm{C}$ complexes. This domain contains the predicted helix domain and a partial extended coiled region in its $\mathrm{C}$-terminus. We further wonder what the N-terminus of CHR functions. Thus, we constructed two plasmids with cpVenus conjugated at CHR's C-terminus (CHRV) and the $\mathrm{N}$-terminus (VCHR) respectively to study the cellular localization of CHR. At first, we checked the location of those proteins in the U2-OS cell line.Since CHR is a transcriptional factor located in the nuclei [13], we predicted these two fused proteins should enter nuclei, too. However, VCHR but not CHRV tended to stay at cytoplasm (Figure 6A). On account of this phenomenon, we hypothesized that a nuclear localization signal (NLS) may lie in the N-terminus of CHR and cpVenus as a rather huge tag (26KD) which can interfere with the NLS function and block the nuclear import. However, although CHR is a nuclear protein, there is no specific sequence predicted as an NLS. Perhaps there is a novel NLS in the N-terminus, so we sought to test whether CHRNV located in the nuclei. We checked the expression of cpVenus fused truncation constructs via immunofluorescence microscopy. In Hela cell line, we found CHRNV was inclined to locate in the nuclei as much as CHRV, while CHRHV and CHRCV spread evenly in whole cell (Figure 6B). Furthermore, we used NIH 3 T3 cells to check distributions of these truncations. Compared with distributions of CHRHV and CHRCV, CHRNV has s strong accumulation in the nuclei (Figure 6C). Noteworthily, the expression of CHRNV was obviously higher than CHRHV and CHRCV in both cell lines, consistent with the western blot results (Figure 3B). Perhaps, the nuclear-entrance protected CHRN from being degraded. Taken together (Figure 6B,C), we suggested that N-terminus of CHR leads itself to enter the nuclei, though we have not identified the exact NLS signal yet.

A
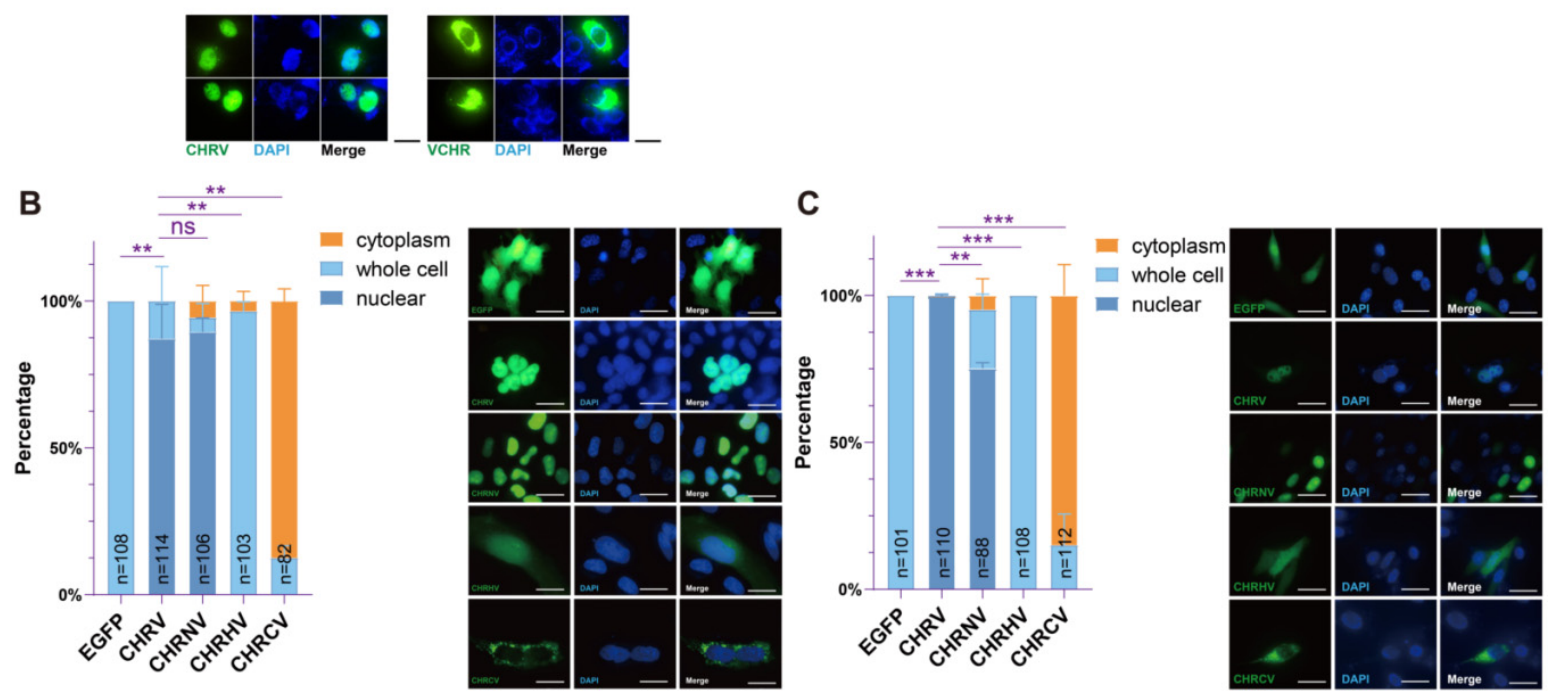

Figure 6. N-terminus of CHR is required for its nuclear localization. (A) Localizations of CHRV (CHR-cpVenus, cpVenus on the C-terminus of CHR) and VCHR (cpVenus-CHR, cpVenus on the $\mathrm{N}$-terminus of CHR) in U2-OS cell line. CHRV is mainly localized in the nuclei, while VCHR mainly stayed in the cytoplasm. Bar $=30 \mu \mathrm{m}$. (B) Different CHR constructs were expressed (right) to study their cellular localizations in Hela cells and analyzed in histogram (left). The nuclear distributions were statistically analyzed with two tailed unpaired t-test. ns, non-significance; ${ }^{* *} p<0.01 .{ }^{* * *} p<0.001$. Bar $=30 \mu \mathrm{m}$. (C) Different CHR constructs were expressed to study their cellular distributions in NIH 3T3 cells (right) and their cellular distributions were statistically analyzed in histogram (left). The nuclear distributions were statistically analyzed with two tailed unpaired t-test. ${ }^{* *} p<0.01$. *** $p<0.001$. Bar $=30 \mu \mathrm{m}$. CHRV was used as a control to show the nuclear localization of CHR, while 
EGFP was used as a control to show universal localization of GFP. According to the data presented in panels (B) and (C), the N-terminus of CHR determines the nuclear localization. Representative images are presented. Examined cell numbers are labeled in each histogram graph.

\section{Discussion}

Here, we showed that a domain of CHR mainly composed of alpha helices can interact with the C-terminus of BMAL1 and repress the transcriptional activity of B/C complexes. Analysis of "ciart" or "chrono" in the Uniprot website suggested that this gene appeared in vertebrate during evolution. No homologous gene has been reported, and no structures have been described. Thus, to learn more about the function/structure relationship of CHR protein, we optimized codon usage to obtain high-yield recombinant proteins (Figure 2A,B), and then performed circular dichroism spectroscopy experiments. CD spectra analysis confirmed predictions of the helical domain and we purified the helical domain of CHR with high yield. We could not crystalize the region which may need more optimization. The N-terminus could help CHR located in the nuclei without any known NLS sequence. Nonetheless, in the CHRN sequences, there are ten arginine and four lysine, which have a potential to compose a novel NLS. Although CHRC had no obvious effects on B/C complex activity, this flexible domain may stabilize the interaction between CHR and BMAL1 or may form complexes with other proteins.

Recently, biochemical analysis and cell-based functional assays have helped us understand how negative factors inhibit the $\mathrm{B} / \mathrm{C}$ complex-dependent transcriptional activation. Duong and colleagues reported that PERs form large complexes with more than ten other proteins, including many known clock proteins BMAL1, CLOCK, CRY1/2, CK1 $/ \varepsilon$, and NONO and other proteins such as PSF, SIN3, and HDAC around the E-box region on chromatin [34]. This large protein complex deacetylates histone 3 and 4 , and then represses B/C complex transcription. In contrast, the molecular PER-PSF-SIN-HDAC axis antagonizes acetyl transferase activity of CLOCK, offering another way that PER2 functions as a repressor [16]. Ye and colleagues found that binding of PERs to the BMAL1/CLOCK/CRY complex removed BMAL1 from the E-box containing promoter regions of ccgs, resulting in loss of the transcriptional activation. Thus, PER proteins could repress transcription via two different ways (Figure 7). The different roles of PER protein may depend on specific proteins PERs interacting with, and more work is required to understand these differences.

CRYs function as a repressor independently of PER proteins. CRY1 is a stronger repressor than CRY2 during negative feedback [22], while CRY2 may repress transcription cooperatively with PER proteins. CRY1 competes with the transcriptional activator, $\mathrm{CBP} / \mathrm{p} 300$ for binding to BMAL1 on chromatin [25]. Early studies identified that the last 43 aa of BMAL1 (632 and 626 aa for mouse and human BMAL1, respectively) are required for association with CRY1 [23], and $\mathrm{Xu}$ and colleagues found that an L606A/L607A mutant at the BMAL1 C-terminus not only disrupted the association between CRY1 and BMAL1, but also abolished circadian cycling [25]. The distal end of BMAL1 is sequestered by CRY1 from transcriptional co-activators $\mathrm{CBP} / \mathrm{p} 300$. Therefore, $\mathrm{CRY} 1$ proteins repress transcription differently from PER proteins.

Using co-IP, we reported that the 579-626 aa region of hBMAL1 is associated with the helical domain of CHR. Unlike previous reports that CHR binds to a different region of BMAL1 from where CRY proteins bind [14], our data supported that CHR and CRY bind to the same TAD domain of BMAL1 (Figure 4). Goriki et al. reported that repression of CHR is HDAC-dependent [13]. Collectively, we proposed a model by which PERs, CRYs, and CHR repress B/C transcriptional complexes (Figure 7). In brief, PER can either form large complexes to recruit HDAC or displace the $\mathrm{B} / \mathrm{C}$ complex away from chromatin; $\mathrm{CRY}$ and CHR interact with the C-terminal end of BMAL1 to block it from transcriptional co-activators; CHR also recruits HDAC to disrupt the activation. PER proteins interact with the PAS domain of BMAL1 and CLOCK proteins [23], and CRY1 and CHR bind to the C-terminus of BMAL1 at different circadian phases (Figure 7). Under such interacting modes, PER, CRY, and CHR differentially act as transcriptional repressors to regulate clock functions. 


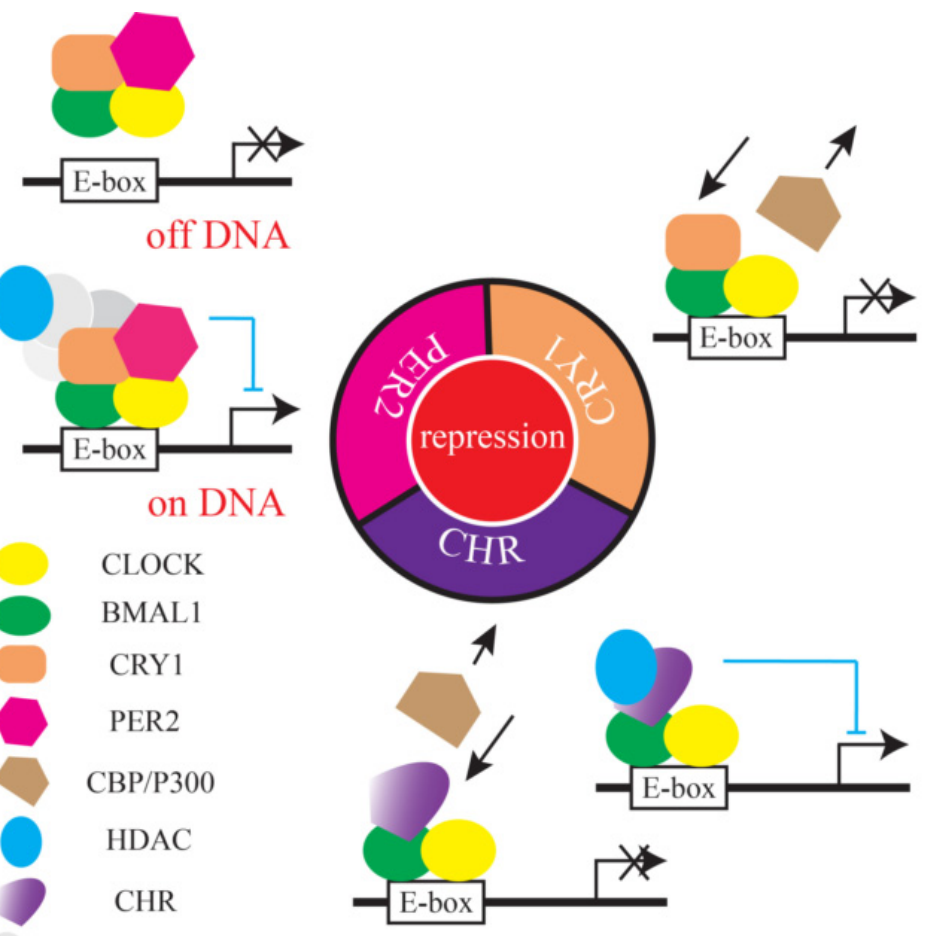

components of PER2 complex

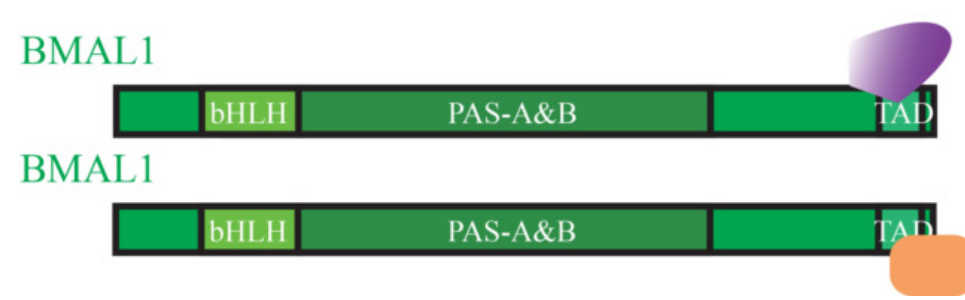

Figure 7. A molecular repression model of how CHR functions as a repressor in the mammalian circadian clock.During repression, PER2 can form large complexes to recruit HDAC (defined in the Abbreviations) or displace the $\mathrm{B} / \mathrm{C}$ complex away from chromatin; $\mathrm{CRY}$ interacts with BMAL1 to restrict it from transcriptional co-activators; CHR binds to BMAL1 to either recruit HDAC or disrupt the recruitment of co-activators. PER proteins associate with the PAS domain of BMAL1; CRY1 binds to the TAD domain in the distal C-terminus of BMAL1.At different circadian phases, CHR binds to the TAD domain in the distal C-terminus of BMAL1. Under these different interacting modes, PER, CRY, and CHR function differentially to repress the circadian clock.

From the model we proposed, it remains undetermined whether CHR can replace CRYs in the mammalian circadian clock. Studies from genetically modified animal models indicate abolished locomotor rhythms in cry1/cry 2 double knockout mice [35,36]. However, SCN slices from neonate cry $1 /$ cry 2 mice had robust circadian luminescence rhythm $[37,38]$. It is conceivable that CHR may compensate for the loss of CRY proteins during early development, but later ciart expressed within specific tissues. More work regarding the expression pattern of CHR, especially in the SCN of cry $1 /$ cry 2 adult knockout mice, may provide important clues to solve this puzzle.

Author Contributions: Y.Y., H.G., and X.Q. conceived and designed research; Y.Y., N.L., and J.Q. performed research; all authors were involved in analyzing data; Y.Y. and X.Q. wrote the paper. All authors have read and agreed to the published version of the manuscript.

Funding: This research was funded by the Anhui Provincial Natural Science Foundation, grant number 1608085MH212 to X.Q. 
Acknowledgments: Ximing Qin acknowledges the start-up fund provided by Anhui University. We are also grateful to the staff for providing technical support with using the facility of Institutes of Physical Science and Information Technology.

Conflicts of Interest: The authors declare no conflict of interest.

\section{Abbreviations}

TTFL transcriptional and translational feedback loop

BMAL1 brain and Muscle Arnt-like protein-1

CLOCK circadian locomotor output cycles kaput

CBP CREB-binding protein

ccg clock controlled genes

ciart circadian associated repressor of transcription

CD circular dichroism

ChIP chromatin Immunoprecipitation

co-IP co-Immunoprecipitation

HDAC histone deacetylase

IF immunofluorescence

CRISPR clustered Regularly Interspaced Short Palindromic Repeat

\section{References}

1. Huang, N.; Chelliah, Y.; Shan, Y.; Taylor, C.A.; Yoo, S.H.; Partch, C.; Green, C.B.; Zhang, H.; Takahashi, J.S. Crystal structure of the heterodimericCLOCK:BMAL1 transcriptional activator complex. Science 2012, 337, 189-194. [CrossRef]

2. Zheng, X.; Sehgal, A. Speed control: Cogs and gears that drive the circadian clock. Trends Neurosci. 2012, 35, 574-585. [CrossRef] [PubMed]

3. Takahashi, J.S. Molecular components of the circadian clock in mammals. Diabetes Obes. Metab. 2015, 17, 6-11. [CrossRef] [PubMed]

4. Kume, K.; Zylka, M.J.; Sriram, S.; Shearman, L.P.; Weaver, D.R.; Jin, X.; Maywood, E.S.; Hastings, M.H.; Reppert, S.M. mCRY1 and mCRY2 are essential components of the negative limb of the circadian clock feedback loop. Cell 1999, 98, 193-205. [CrossRef]

5. Hardin, P.E.; Panda, S. Circadian timekeeping and output mechanisms in animals. Curr. Opin. Neurobiol. 2013, 23, 724-731. [CrossRef] [PubMed]

6. Preitner, N.; Damiola, F.; Lopez-Molina, L.; Zakany, J.; Duboule, D.; Albrecht, U.; Schibler, U. The orphan nuclear receptor REV-ERBalpha controls circadian transcription within the positive limb of the mammalian circadian oscillator. Cell 2002, 110, 251-260. [CrossRef]

7. Sato, T.K.; Panda, S.; Miraglia, L.J.; Reyes, T.M.; Rudic, R.D.; McNamara, P.; Naik, K.A.; FitzGerald, G.A.; Kay, S.A.; Hogenesch, J.B. A functional genomics strategy reveals Rora as a component of the mammalian circadian clock. Neuron 2004, 43, 527-537. [CrossRef]

8. Eide, E.J.; Woolf, M.F.; Kang, H.; Woolf, P.; Hurst, W.; Camacho, F.; Vielhaber, E.L.; Giovanni, A.; Virshup, D.M. Control of mammalian circadian rhythm by CKIepsilon-regulated proteasome-mediated PER2 degradation. Mol. Cell. Biol. 2005, 25, 2795-2807. [CrossRef]

9. Maywood, E.S.; Chesham, J.E.; Smyllie, N.J.; Hastings, M.H. The Tau mutation of casein kinase $1 \varepsilon$ sets the period of the mammalian pacemaker via regulation of Period1 or Period 2 clock proteins. J. Biol. Rhythms 2014, 29, 110-118. [CrossRef]

10. Hirano, A.; Yumimoto, K.; Tsunematsu, R.; Matsumoto, M.; Oyama, M.; Kozuka-Hata, H.; Nakagawa, T.; Lanjakornsiripan, D.; Nakayama, K.I.; Fukada, Y. FBXL21 regulates oscillation of the circadian clock through ubiquitination and stabilization of cryptochromes. Cell 2013, 152, 1106-1118. [CrossRef]

11. Yoo, S.H.; Mohawk, J.A.; Siepka, S.M.; Shan, Y.; Huh, S.K.; Hong, H.K.; Kornblum, I.; Kumar, V.; Koike, N.; $\mathrm{Xu}, \mathrm{M}$;; et al. Competing E3 ubiquitin ligases govern circadian periodicity by degradation of CRY in nucleus and cytoplasm. Cell 2013, 152, 1091-1105. [CrossRef] [PubMed] 
12. Meng, Q.J.; Maywood, E.S.; Bechtold, D.A.; Lu, W.Q.; Li, J.; Gibbs, J.E.; Dupré, S.M.; Chesham, J.E.; Rajamohan, F.; Knafels, J.; et al. Entrainment of disrupted circadian behavior through inhibition of casein kinase 1 (CK1) enzymes. Proc. Natl. Acad. Sci. USA 2010, 107, 15240-15245. [CrossRef] [PubMed]

13. Goriki, A.; Hatanaka, F.; Myung, J.; Kim, J.K.; Yoritaka, T.; Tanoue, S.; Abe, T.; Kiyonari, H.; Fujimoto, K.; Kato, Y.; et al. A novel protein, $\mathrm{CHRONO}$, functions as a core component of the mammalian circadian clock. PLoS Biol. 2014, 12, e1001839. [CrossRef] [PubMed]

14. Anafi, R.C.; Lee, Y.; Sato, T.K.; Venkataraman, A.; Ramanathan, C.; Kavakli, I.H.; Hughes, M.E.; Baggs, J.E.; Growe, J.; Liu, A.C.; et al. Machine learning helps identify CHRONO as a circadian clock component. PLoS Biol. 2014, 12, e1001840. [CrossRef]

15. Annayev, Y.; Adar, S.; Chiou, Y.Y.; Lieb, J.D.; Sancar, A.; Ye, R. Gene model 129 (Gm129) encodes a novel transcriptional repressor that modulates circadian gene expression. J. Biol. Chem. 2014, 289, 5013-5024. [CrossRef]

16. Ye, R.; Selby, C.P.; Chiou, Y.Y.; Ozkan-Dagliyan, I.; Gaddameedhi, S.; Sancar, A. Dual modes of CLOCK:BMAL1 inhibition mediated by Cryptochrome and Period proteins in the mammalian circadian clock. Genes Dev. 2014, 28, 1989-1998. [CrossRef]

17. Chiou, Y.Y.; Yang, Y.; Rashid, N.; Ye, R.; Selby, C.P.; Sancar, A. Mammalian Period represses and de-represses transcription by displacing CLOCK-BMAL1 from promoters in a Cryptochrome-dependent manner. Proc. Natl. Acad. Sci. USA 2016, 113, E6072-E6079. [CrossRef]

18. Koike, K.; Yoo, S.H.; Huang, H.C.; Kumar, V.; Lee, C.; Kim, T.K.; Takahashi, J.S. Transcriptional architecture and chromatin landscape of the core circadian clock in mammals. Science 2012, 338, 349-354. [CrossRef]

19. Duong, H.A.; Weitz, C.J. Temporal orchestration of repressive chromatin modifiers by circadian clock Period complexes. Nat. Struct. Mol. Biol. 2014, 21, 126-132. [CrossRef]

20. Langmesser, S.; Tallone, T.; Bordon, A.; Rusconi, S.; Albrecht, U. Interaction of circadian clock proteins PER2 and CRY with BMAL1 and CLOCK. BMC Mol. Biol. 2008, 9, 41. [CrossRef]

21. Ye, R.; Selby, C.P.; Ozturk, N.; Annayev, Y.; Sancar, A. Biochemical analysis of the canonical model for the mammalian circadian clock. J. Biol. Chem. 2011, 286, 25891-25902. [CrossRef] [PubMed]

22. Khan, S.K.; Xu, H.; Ukai-Tadenuma, M.; Burton, B.; Wang, Y.; Ueda, H.R.; Liu, A.C. Identification of a novel cryptochrome differentiating domain required for feedback repression in circadian clock function. J. Biol. Chem. 2012, 287, 25917-25926. [CrossRef] [PubMed]

23. Xu, H.; Gustafson, C.L.; Sammons, P.J.; Khan, S.K.; Parsley, N.C.; Ramanathan, C.; Lee, H.W.; Liu, A.C.; Partch, C.L. Cryptochrome 1 regulates the circadian clock through dynamic interactions with the BMAL1 C terminus. Nat. Struct. Mol. Biol. 2015, 22, 476-484. [CrossRef] [PubMed]

24. Michael, A.K.; Fribourgh, J.L.; Chelliah, Y.; Sandate, C.R.; Hura, G.L.; Schneidman-Duhovny, D.; Tripathi, S.M.; Takahashi, J.S.; Partch, C.L. Formation of a repressive complex in the mammalian circadian clock is mediated by the secondary pocket of CRY1. Proc. Natl. Acad. Sci. USA 2017, 114, 1560-1565. [CrossRef]

25. Kiyohara, Y.B.; Tagao, S.; Tamanini, F.; Morita, A.; Sugisawa, Y.; Yasuda, M.; Yamanaka, I.; Ueda, H.R.; van der Horst, G.T.; Kondo, T.; et al. The BMAL1 C terminus regulates the circadian transcription feedback loop. Proc. Natl. Acad. Sci. USA 2006, 103, 10074-10079. [CrossRef]

26. Yamazaki, S.; Takahashi, J.S. Real-time luminescence reporting of circadian gene expression in mammals. Methods Enzymol. 2005, 393, 288-301.

27. Yachdav, G.; Kloppmann, E.; Kajan, L.; Hecht, M.; Goldberg, T.; Hamp, T.; Hönigschmid, P.; Schafferhans, A.; Roos, M.; Bernhofer, M.; et al. PredictProtein-an open resource for online prediction of protein structural and functional features. Nucleic Acids Res. 2014, 42, W337-W343. [CrossRef]

28. Jones, D.T. Protein secondary structure prediction based on position-specific scoring matrices. J. Mol. Biol. 1999, 292, 195-202. [CrossRef]

29. Cheng, M.Y.; Bullock, C.M.; Li, C.; Lee, A.G.; Bermak, J.C.; Belluzzi, J.; Weaver, D.R.; Leslie, F.M.; Zhou, Q.Y. Prokineticin 2 transmits the behavioural circadian rhythm of the suprachiasmatic nucleus. Nature 2002, 417, 405-410. [CrossRef]

30. Andrade, M.A.; Chacón, P.; Merelo, J.J.; Morán, F. Evaluation of secondary structure of proteins from UV circular dichroism spectra using an unsupervised learning neural network. Protein Eng. 1993, 6, 383-390. [CrossRef] 
31. Czarna, A.; Breitkreuz, H.; Mahrenholz, C.C.; Arens, J.; Strauss, H.M.; Wolf, E. Quantitative analyses of cryptochrome-mBMAL1 interactions: Mechanistic insights into the transcriptional regulation of the mammalian circadian clock. J. Biol. Chem. 2011, 286, 22414-22425. [CrossRef] [PubMed]

32. Takahata, S.; Ozaki, T.; Mimura, J.; Kikuchi, Y.; Sogawa, K.; Fujii-Kuriyama, Y. Transactivation mechanisms of mouse clock transcription factors, mClock and mArnt3. Genes Cells 2000, 5, 739-747. [CrossRef] [PubMed]

33. Cong, L.; Ran, F.A.; Cox, D.; Lin, S.; Barretto, R.; Habib, N.; Hsu, P.D.; Wu, X.; Jiang, W.; Marraffini, L.A.; et al. Multiplex genome engineering using CRISPR/Cas systems. Science 2013, 339, 819-823. [CrossRef]

34. Duong, H.A.; Robles, M.S.; Knutti, D.; Weitz, C.J. A molecular mechanism for circadian clock negative feedback. Science 2011, 332, 1436-1439. [CrossRef]

35. van der Horst, G.T.; Muijtjens, M.; Kobayashi, K.; Takano, R.; Kanno, S.; Takao, M.; de Wit, J.; Verkerk, A.; Eker, A.P.; van Leenen, D.; et al. Mammalian Cry1 and Cry2 are essential for maintenance of circadian rhythms. Nature 1999, 398, 627-630. [CrossRef]

36. Vitaterna, M.H.; Selby, C.P.; Todo, T.; Niwa, H.; Thompson, C.; Fruechte, E.M.; Hitomi, K.; Thresher, R.J.; Ishikawa, T.; Miyazaki, J.; et al. Differential regulation of mammalian period genes and circadian rhythmicity by cryptochromes 1 and 2. Proc. Natl. Acad. Sci. USA 1999, 96, 12114-12119. [CrossRef] [PubMed]

37. Ono, D.; Honma, S.; Honma, K. Differential roles of AVP and VIP signaling in the postnatal changes of neural networks for coherent circadian rhythms in the SCN. Sci. Adv. 2016, 2, e1600960. [CrossRef]

38. Ono, D.; Honma, S.; Honma, K. Cryptochromes are critical for the development of coherent circadian rhythms in the mouse suprachiasmatic nucleus. Nat. Commun. 2013, 4, 1666. [CrossRef]

(C) 2020 by the authors. Licensee MDPI, Basel, Switzerland. This article is an open access article distributed under the terms and conditions of the Creative Commons Attribution (CC BY) license (http://creativecommons.org/licenses/by/4.0/). 